\title{
ELECTRON RADIATION BELT DYNAMICS DURING MAGNETIC STORMS AND IN QUIET TIME
}

\section{L.L. Lazutin}

Scobeltsyn Institute of Nuclear Physics, Moscow State University

Moscow,Russia, lll@srd.sinp.msu.ru, l.l.lazutin@gmail.com

\section{A.V. Dmitriev}

Scobeltsyn Institute of Nuclear Physics,

Moscow State University

Moscow, Russia,dalex@jupiter.ss.ncu.edu.tw

\section{A.V. Suvorova}

Scobeltsyn Institute of Nuclear Physics, Moscow State University

Moscow,Russia,all@yahoo.com
Abstract. The paper discusses the outer electron belt dynamics, adiabatic and nonadiabatic mechanisms of increases and losses of energetic electrons.

Under undisturbed conditions, the outer electron belt gradually empties: in the inner magnetosphere due to electron losses in the atmosphere and in the quasitrapping region due to losses at the magnetopause because drift shells of electrons are not closed there. The latter process does not occur in normal years due to the masking replenishment by freshly accelerated particles, but in years of extremely low activity it leads to a significant decrease in the electron population of the belt.

During the magnetic storm main phase, the first reason for the decrease in the electron flux intensity is the adiabatic cooling associated with conservation of adiabatic invariants and complemented by injection of electrons into the atmosphere and their losses at the magnetopause. Electron flux increases involve $\mathbf{E} \times \mathbf{B}$ electron injection by the induction electric field of substorm ac- tivation and by the large-scale solar wind electric field, with pitch energy diffusion along with adiabatic heating in the recovery phase.

The rate of electron flux recovery after a storm is determined by the ratio of nonadiabatic increases and losses; hence the electron flux represents a continuous series from low to very high values. The combination of these processes determines the individual character of radiation belt development during each magnetic storm and the behavior of the belt in the quiet time.

Keywords: magnetosphere, electrons, radiation belt, replenishment and losses.

\section{INTRODUCTION}

Existing reviews on radiation belts (RB) [Parks, Winkler 1968; Vernov et al., 1969; Friedel et al., 2002; Millan, Thorne, 2007; Shprits et al., 2008a] describe in sufficient detail both the structure of $\mathrm{RB}$ and its formation. According to the traditional theory [Tverskoy, 1964, 1965], the RB formation is attributed to the combination of slow radial diffusion of electrons driven by small magnetic field pulses with losses in the atmosphere due to pitch-angle diffusion. In principle, this explains the observed spatial structure of trapped electrons with a slot region between inner and outer radiation belts.

During magnetic storms, this pattern is disturbed; there occur dynamic variations, decreases, and increases in energetic electron fluxes. Fluxes of energetic "killer" electrons attract greater attention of researchers, there are works devoted to the prediction of their mean or maximum values [Li et al., 2001; Simms et al., 2016] (see also the review [Potapov, 2017]).

A large number of papers analyze the $\mathrm{RB}$ dynamics during magnetic storms [Baker et al., 1997; Li et al., 1997; Reeves et al., 1998; Yu et al., 2015; Hwang et al., 2015; Turner et al., 2017]; a lot of these studies have been carried out at SINP MSU [Vernov et al., 1965; Kuznetsov et al., 1966; Bakhareva, 2003; Ivanova et al.,
2000; Kalegaev et al., 2015; Antonova, 2005; Lazutin, 2012; Kalegaev, Vlasova, 2014; Dmitriev et al., 2010, 2014; Slivka et al., 2006; Tverskaya et al., 2005; Vernov et al., 1969]. Accordingly, it seems appropriate to review current ideas about the RB dynamics. Note that the review is based largely on studies carried out at SINP MSU.

In the review, we first describe the RB dynamics during magnetic storms and then the mechanisms of replenishment and losses of energetic electrons; finally, we examine the behavior of RB in the quiet time. This paper is intended for use by readers familiar with the basic concepts: particle motion and adiabatic invariants, dynamics of fields and currents during magnetic storms, etc.

\section{MAGNETIC STORMS}

Figure 1 shows energetic electron and proton fluxes measured by the low-orbit satellite SERVIS from February 5 to February 25, 2004, in particular during the February 11, 2004 magnetic storm. Flux intensity dips are observed in all storms on all RB shells in a wide range of energies and for particles of different types. These dips are largely caused by a change in the structure of the magnetosphere when subject to the so-called 


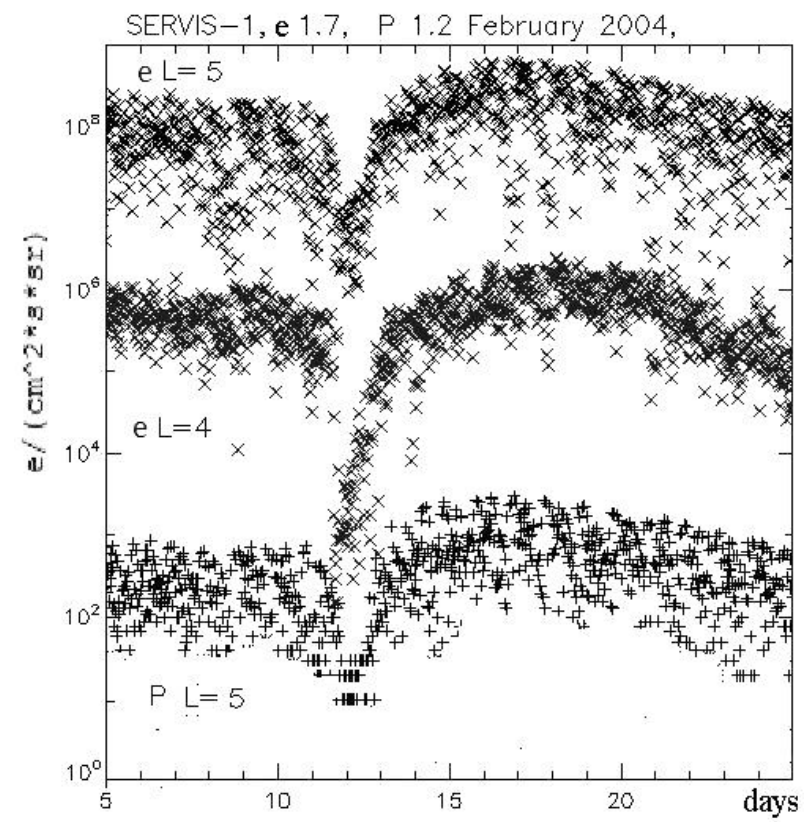

Figure 1. Electron and proton fluxes measured by SERVIS-1 when it passes over the $L$ levels in the Northern Hemisphere on February 5-25, 2004. A magnetic storm with a minimum Dst $=-110$ nT occurred on February 11, 2004

Dst effect. This effect is necessarily present in the storm main phase, but there may be additional nonadiabatic losses, in particular precipitation of particles into the atmosphere. If there are no nonadiabatic processes (losses or accelerations), the RB configuration and particle fluxes should fully recover after a storm.

But often this does not happen. After a storm, the electron flux can return to the pre-storm level as well as exceed it or not recover completely. In the wellknown paper [Reeves et al., 2003], storms are divided into these three types. In fact, this division is arbitrary (see Figure 3, $a, c$ in [Reeves et al., 2003]). If we do not pay attention to the difference in the marks assigned by the author, after the storm there is a continuous series of electron flux intensities lower or higher than prestorm ones. We think that this series is created by different ratios of particle flux losses and increases during the storm: if the losses exceed replenishment, the flux is not fully recovered, if they do not exceed replenishment, the flux after the storm is higher than the pre-storm one.

\section{ADIABATIC VARIATIONS (Dst EFFECT)}

During the magnetic storm main phase, the magnetic field strength in internal field lines decreases. To maintain the same magnetic flux level, the drift shell shifts outward to the region where field lines are longer; therefore, to conserve the second invariant (the distance between mirror points), mirror points are displaced along a field line upward. A decrease in the magnetic field strength at the new, higher mirror point leads to a decrease in particle energy because the first adiabatic invariant (magnetic moment) should remain unchanged.

This effect, first described by McIlwain [1966] and later by Kim and Chan [1997], is called the Dst effect. During the storm main phase, the Dst effect always caus- es a decrease in the intensity of electron fluxes, which serves as a base for additional nonadiabatic effects.

During the storm recovery phase, an adiabatic return to the pre-storm level should occur; however, the loss and acceleration processes decrease or increase the number of particles involved in the recovery process, thus producing a continuous series of intensity of electron fluxes.

Note that a rise of solar wind pressure in the storm initial phase, which leads to an increase in the magnetic field intensity rather than its decrease, as in the storm main phase, causes an inverse Dst effect — an adiabatic increase in the energy of trapped magnetic particles and thus an increase in the intensity of their fluxes. A part of particles close to the loss cone due to the decrease in the position of mirror points precipitates into the atmosphere and is lost there, but a satellite in RB does not register this loss.

\section{ADIABATIC VARIATIONS. DAWN-DUSK ASYMMETRY}

When studying time variations in electron flux intensities during a storm, a false impression of disappearance of particles may be created when we, instead of the magnetic drift trajectory varying during the storm, use the $L$ coordinate of undisturbed level, which is usually given in a database of measurements from low-orbit satellites.

Our Figure 1 (as in many Figures from other papers) uses $L$ as an indicator of the normalized distance to the top of a field line, but it is improper to use the undisturbed level parameter $L$ to describe the particle dynamics during a magnetic storm. Let us illustrate this statement by a concrete example.

At the beginning of the storm main phase, the ring current asymmetry creates a situation where the electron flux on the dusk side decreases, while the dawn flux remains undisturbed (Figure 2). In [Lazutin, 2016], this effect has received a simple explanation: the drift shell in the attenuated dusk magnetic field is adiabatically displaced to lower latitudes. Since many Figures represent electron fluxes as a function of the $L$ coordinate calculated for the quiet magnetic activity level and not for the corresponding moments of the storm, we can consider the presented picture as a result of the displacement of the drift shell toward Earth.

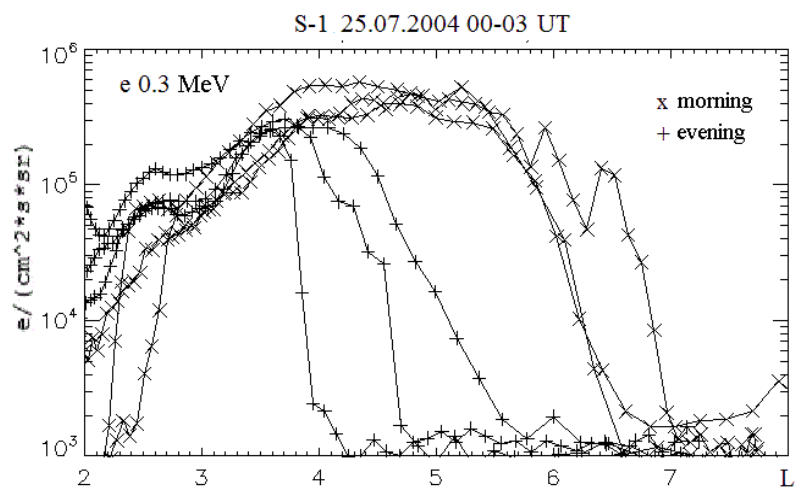

Figure 2. Six consecutive electron profiles recorded by SERVIS- 1 on the dawn and dusk sides in the $0.3 \mathrm{MeV}$ channel during the main phase of the July 25, 2004 magnetic storm 


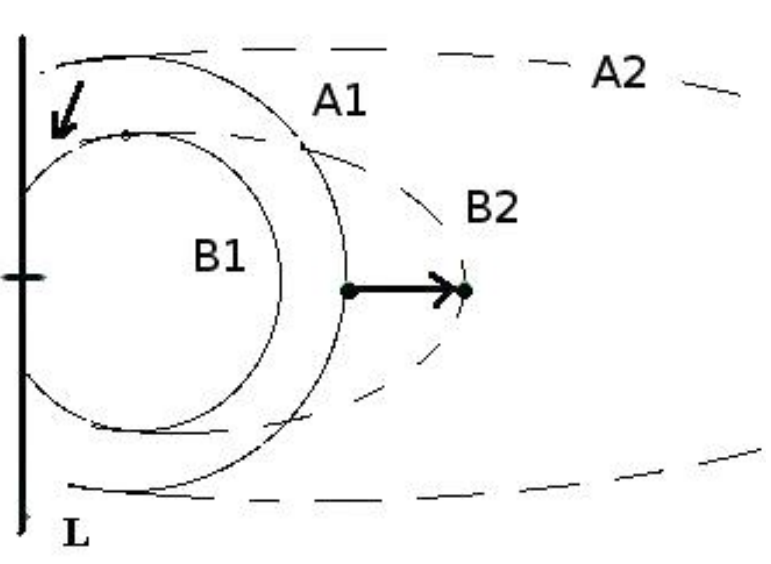

Figure 3. During the storm main phase, the field lines A1 and B1 are transformed into A2 and B2. Drift shells of particles go (along arrows) from A1 to B2; at the equator, from Earth; and near footpoints of field lines, to lower latitudes

There is an apparent contradiction: due to the Dst effect, the drift shell goes away from Earth, whereas according to the said considerations it approaches Earth. Are these two different adiabatic effects? But this should not be so. An explanation of the contradiction is given in Figure 3. The change in the magnetic field during the storm main phase actually shifts the drift shell at the equator from Earth, but at low heights, near the footpoint of the field line the drift shell is displaced to lower latitudes because of its new shape.

Let us examine mechanisms of losses and accelerations working during a storm. We start with the interaction with waves that can cause both a decrease and an increase in the energetic electron flux.

\section{INTERACTION OF ELECTRONS WITH HIGH-FREQUENCY WAVES}

Energetic electrons can effectively interact with plasmaspheric hisses and with VLF emission above the plasmapause. The interaction leads to diffusion both along pitch angles and in the velocity space, thus causing precipitation of particles into the atmosphere and a decrease in flux intensity as well as particle acceleration and an increase in flux intensity and electron energy. Gyroresonance with waves of various types in the range from 0.1 to units of electron gyrofrequency described in [Horne, Thorne, 1998; 2003] has also been extensively experimentally and theoretically studied.

Changes in particle energy and pitch angle under the impact of resonance with trains of VLF emission cause the particle to wander along pitch angles, thus leading to its precipitation into the loss cone and dropout in the atmosphere. The electron dropout in the atmosphere during the interaction with VLF emission has been studied in a number of theoretical papers (for example, [Shprits et al., 2008b]) and is described in detail in studies based on measurements; in some of them, their dropouts in the atmosphere are considered as the main mechanism causing an electron flux decrease after a storm [Zakharov, Kuznetsov, 1978; Summers et al., 2004; Meredith et al., 2007; Xiao et al., 2014].
Other articles demonstrate the significance of pitchangle diffusion in velocities for electron flux increases (Summers et al., 1998; Horne et al., 2005; Demekhov et al., 2006; Foster et al., 2017]. Acceleration to energies of the order of $1 \mathrm{MeV}$ occurs in $\mathrm{RB}$ with a maximum at $L \sim 4-7$. The condition for the effective increase in the electron flux is a high substorm activity, which produces freshly accelerated electrons with a 20-100 keV energy serving as the initial flux; and a high power of chorus is observed.

Relativistic electrons are accelerated due to parasitic diffusion by waves generated by less energetic electrons. Such two-stage acceleration is described, for example, in [Jaynes et al., 2015; Foster et al., 2017].

According to [Summers et al., 2004], the characteristic time of acceleration and losses is approximately the same and is about a day, but it varies greatly depending on parameters of a medium.

Exotic, but real process is the electron precipitation due to parasitic resonance with electromagnetic ioncyclotron (EMIC) waves [Summers, Thorne, 2003; Lazutin et al., 2011; Ni et al., 2015; Kubota, Omura, 2017]. EMIC waves are generated by ring current protons during the storm main phase. Since the cyclotron frequency of protons is much lower than the cyclotron frequency of electrons, the interaction occurs as a result of Doppler frequency shift in the opposing wave and particle motion. In this case, the longitudinal electron velocity should be very high, and hence it causes precipitation of electrons only with an energy of several $\mathrm{MeV}$.

\section{LOSSES AT THE MAGNETOPAUSE}

Sufficiently convincing arguments for the mechanism of electron dropout at the magnetopause have been put forward in [Dmitriev, Chao, 2003; Shprits et al., 2006, Millan, Thorne, 2007; Kim et al., 2008; Saito et al., 2010; Matsumura et al., 2011; Turner et al., 2012; Hudson et al., 2014; Lazutin, 2016]. In the quasitrapping region, drift shells are not closed; on the night side, electrons drift with adiabatic invariants remaining unchanged to the morning magnetosphere-magnetopause boundary; protons, to the evening boundary (and vice versa on the dayside); then they go from the closed field lines to the turbulent magnetosheath. This continuous outflow of electrons from RB increases during the storm main phase, when the Dst effect shifts drift shells of electrons from Earth [Reeves et al., 2003]. Moreover, magnetic storms often occur with sharp increases in solar wind plasma density and velocity, causing an abrupt shift of the magnetopause to Earth and an increase in losses of electrons from the outer magnetosphere [Dmitriev, Chao, 2003].

Due to the pitch-angle diffusion, a small part of electrons (a few percent) can return from the magnetosheath to drift orbits, as evidenced by the so-called drift echo effect [Vampola, Korth, 1992], but most of the particles go to the magnetotail [Daibog et al., 2015] and to interplanetary space.

As a result, the quasi-trapping region should quickly empty; however, as can be seen from measurements made at the geostationary orbit (see, for example, 
Electrons $>0.6 \& 2.0 \mathrm{MeV}, 2009$

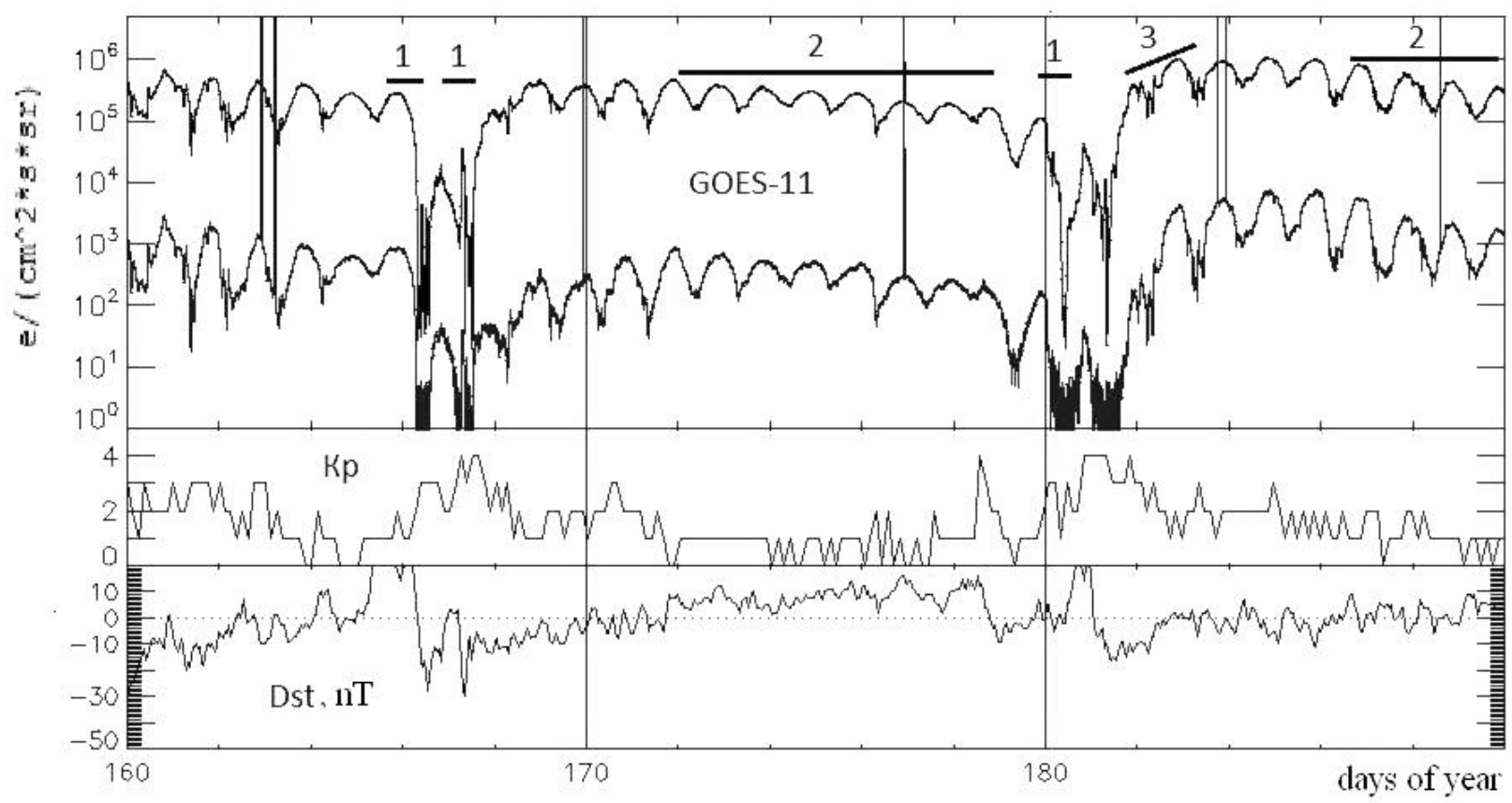

Figure 4. Electron fluxes and magnetic activity indices (moderate disturbances): "sinusoidal” electron flux variations manifestation of the day-night asymmetry of the magnetospheric magnetic field; 1 - an electron flux decrease during the storm main phase, 2 - electron losses at the magnetopause, 3 - an electron flux increase due to radial drift from the center of RB

Figure 4), it does not empty due primarily to the oncoming acceleration; and in the absence thereof, due to the diffusion of particles from the region of radiation belt maximum (a region closer to Earth), which is opposite to the radial diffusion from Earth. Radial diffusion from a region with a high particle flux into a region with a lower one is described, for example, in [Shprits et al., 2006]. As a result, the electron density in RB decreases if, of course, there is no replenishment due to particle acceleration. Intervals of slow decrease in the intensity of electron fluxes are shown in Figure 4; in these time intervals the $K_{\mathrm{p}}$ index is low, the substorm activity is absent. The rest of the time, the replenishment of electron population due to substorm activity masks this process.

\section{ACCELERATION PROCESSES. SLOW E×B DRIFT}

There is a wide variety of mechanisms for accelerating energetic electrons in the magnetosphere. We have already said that electrons can accelerate at resonance with VLF waves. Along with the cyclotron resonance, we can expect a drift resonance with magnetospheric Pc5 pulsations or solar wind pulsations with a period of the order of several minutes. Acceleration of this type is mentioned, say, in [Elkington et al., 1999; Ukhorskiy et al., 2006]. The acceleration of electrons due to the radial electric drift or their shift to the region of a stronger magnetic field is faster and more efficient. A large-scale electric field is driven by the solar wind. The Akasofu index representing the effectiveness of the solar wind effect on Earth's magnetosphere contains the velocity

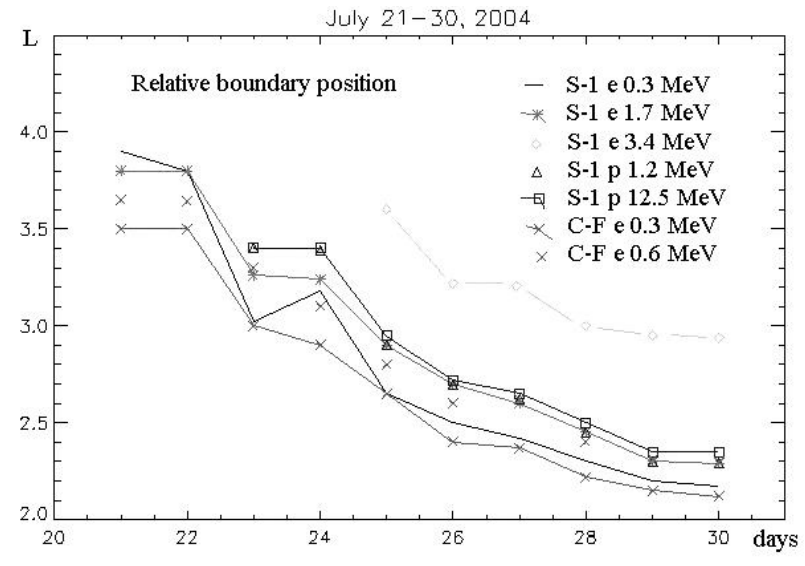

Figure 5. Earthward shift of the low-latitude slope of RB during the chain of magnetic storms in July 2004

and magnitude of the vertical component of the interplanetary magnetic field.

A dawn-to-dusk large-scale convective electric field spins up the convective vortex in the magnetotail. This field penetrates both into the auroral magnetosphere and into the outer $\mathrm{RB}$, thus allowing $\mathrm{RB}$ particles to be accelerated by the electric drift, which is well documented [Tverskaya et al., 2005; Califf et al., 2017; Claudepierre et al., 2017; Millan, Baker, 2012; Turner et al., 2017]. Califf et al. [2017] state that the $\mathbf{E} \times \mathbf{B}$ drift is effective up to $500 \mathrm{keV}$, whereas for high-energy electrons we have to take into account additional mechanisms. Indeed, the authors consider electrons of the plasma layer in the magnetotail to be the initial source; energy of the electrons is two orders of magnitude lower than that of the substorm auroral electrons. Especially powerful 


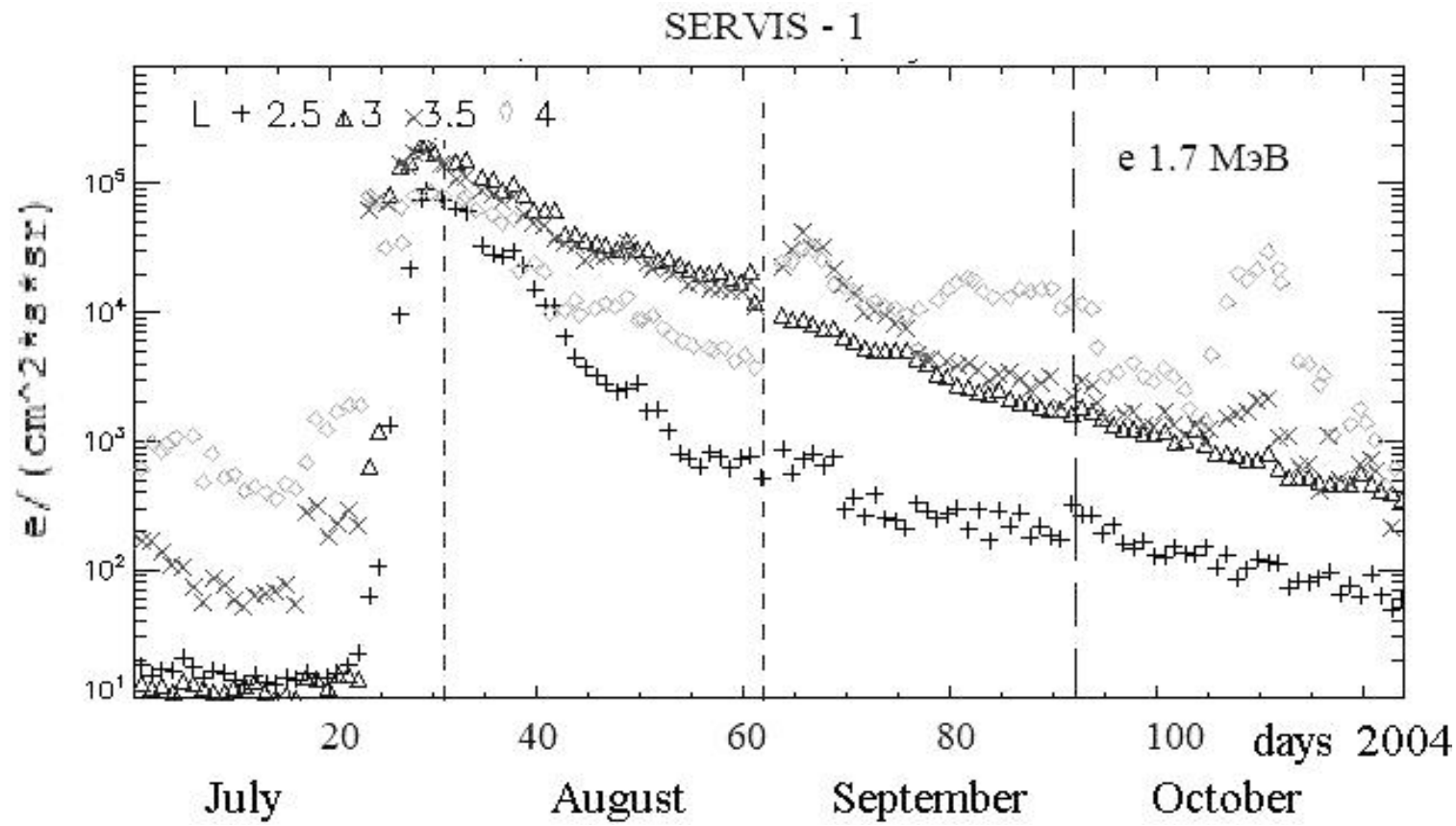

Figure 6. Relaxation of excess electron flux after the July 2004 storms

acceleration and the increased particle flux remaining unchanged for many days are observed as a result of chains of successive magnetic storms. Such an unusual case is described in [Kuznetsov et al., 2009; Yang et al., 2014]. Using data from the former paper, we show in Figure 5 how the low-latitude boundary of electrons and protons, trapped in $\mathrm{RB}$, shifts (those days, $\mathrm{RB}$ was filled with solar protons). The earthward shift is driven by the $\mathbf{E} \times \mathbf{B}$ drift. This is confirmed by the equal velocity for particles of all energies and types.

Moving to the region of higher magnetic field strength, particles increase their energy. This causes an increase in the particle flux. Figure 6 [Lazutin et al., 2012] shows that RB gets rid of such an overpopulation for a long time. Note that strong storms may result in the formation of additional energetic electron belts in the vicinity of the slot region, which also exist for months [Vernov et al., 1965; Kuznetsov, 1966; Logachev, Lazutin, 2012].

\section{ACCELERATION PROCESSES. FAST E $\times$ B SHIFT}

An electric field can also be short-term, caused by a rapid change in the magnetic field at the time of SC or substorm activation. The shift of electrons and protons by an SC pulse deep into RB, detected during measurements made by CRRES [Li et al., 1993] on March 24, 1991, was analyzed in [Pavlov et al., 1993]. The SC pulse is double - a positive pulse followed by a negative one; therefore, if particles are not transferred by the magnetic drift to the night side on time, an increase in their energy (and flux intensity) is eliminated adiabatically. The particles which have a 1-3 min half-period of drift pass to the night side, where they also experience a radial drift with acceleration driven by a negative pulse. We can thus estimate the lower limit for particle ener- gies for which the mechanism will operate - on the order of a few $\mathrm{MeV}$. If we consider that high SC pulses (from $50 \mathrm{nT}$ and higher) occur at most twice a year, the shift of electrons with an energy of several $\mathrm{MeV}$ by an SC pulse to a greater depth does not play an important role in RB dynamics.

Much more often there is a pulsed dipolization of the magnetic field at the moment of breakup or re-activation of a substorm. It leads to the fact that along with the increase in the flux of low-energy (to several keV) electrons, responsible for auroras and accelerated by a longitudinal electric field, there is an impulsive increase in the flux of energetic electrons in the auroral magnetosphere. Characteristics of these increases have been extensively studied using both balloon [Anderson, 1965] and satellite observations, in particular CREES measurements in the equatorial plane. As a rule, these electrons appear in a quasi-trapping region, have an energy 20-200 keV, and cause subsequent acceleration by the radial $\mathbf{E} \times \mathbf{B}$ drift, mentioned above [Tang et al., 2016].

At the same time, during magnetic storms when the region of substorm activations shifts to Earth, the substorm electric field pulse shifts electrons to the center of $\mathrm{RB}$, to the region of high magnetic field strength, and the ultimate energy of freshly accelerated electrons can reach several MeV [Lazutin, 2013]. Consequently, the electron flux gradient along the outer RB slope increases; and in the quasi-trapping region the particle flux gradually increases due to the outward drift. The electron acceleration via the substorm shift has also been considered in [Kabin et al., 2017; Gabrielse et al., 2016, 2017].

Nishida [1976] has put forward an idea of electron "recirculation" according to which electrons, once accelerated by electric drift, move again to a distant field line and are accelerated in this way many times. The idea suggested for Jupiter's magnetosphere has found 


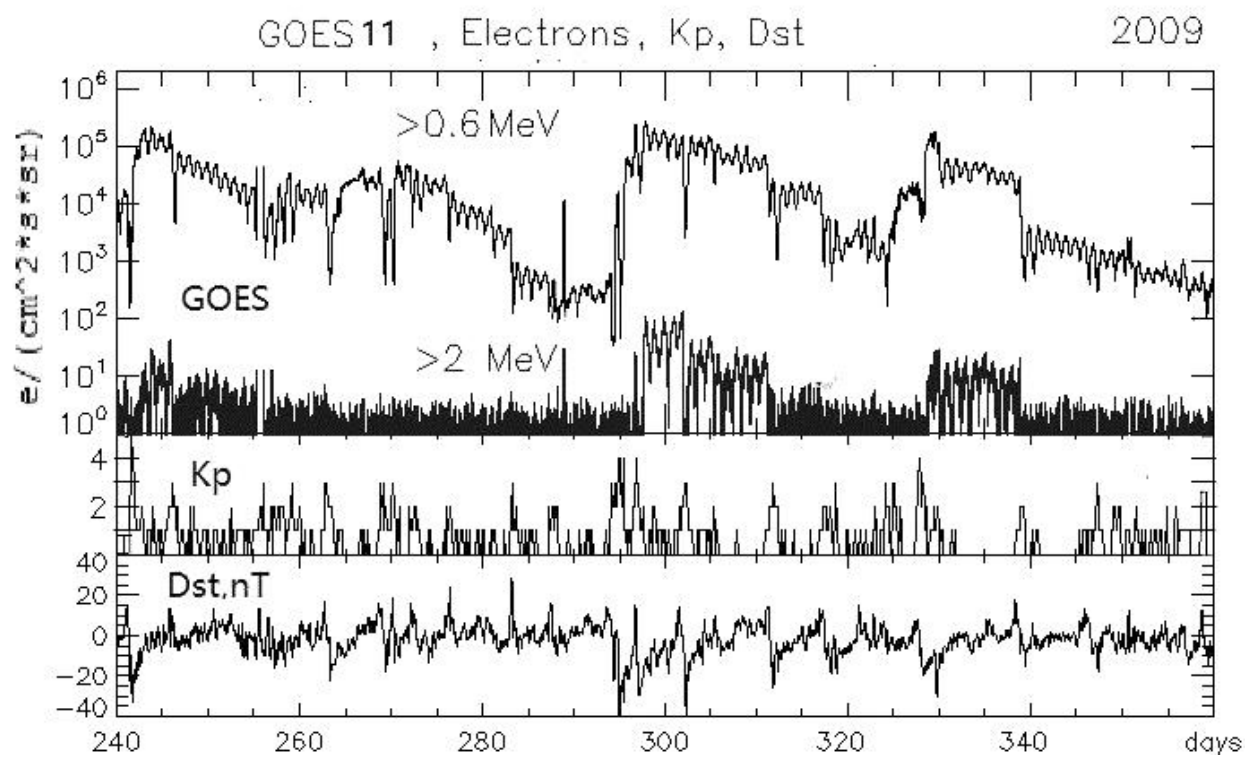

Figure7. Electron flux in two energy channels, measured by the geostationary satellite GOES-11, and magnetic activity indices (the last quarter of 2009)

supporters who applied it to Earth's magnetosphere [Baker et al., 1997].

Zhenpeng Su et al. [2014] emphasize the difference between subrelativistic $(\sim 0.1 \mathrm{MeV})$ and relativistic (2-5 MeV) electrons: the former provide up to $100 \%$ of flux increases due to the shift deep into RB; the latter, only $20 \%$; in this case, the increase takes a few minutes. According to the calculation, the interaction with choruses gives $60-80 \%$ of increases in the flux of energetic electrons (0.2-5.0 MeV), which take a longer time ( $6 \mathrm{hr})$. A similar difference in the behavior of electrons of different energies has been revealed in the statistical analysis carried out by Ying Xiong et al. [2015]: the flux of $0.3-2.5 \mathrm{MeV}$ energetic electrons increases in $87 \%$ of storms, whereas the flux of $2.5-14 \mathrm{MeV}$ electrons, only in $35 \%$ of storms.

\section{RADIAL TRANSPORT}

Radial transport, shift, injection, diffusion - there are many terms and mechanisms; and not to get confused and not to confuse readers, we briefly describe these mechanisms.

To Earth, a particle flux is transported with acceleration by pulsed substorm injection and $\mathbf{E} \times \mathbf{B}$ drift in a large-scale electric field during a magnetic storm, by wave and pulsed radial diffusion. The last mechanism (the slowest according to [Tverskoy, 2004]) is responsible for the formation of the electron radiation belt.

At the end of the storm main phase there occurs a returned adiabatic transport to Earth.

Moreover, both during the storm and during the quiescent period, a continuous electron flux from Earth is observed as a result of drift due to the negative radial gradient of trapped particles.

\section{THE QUIET MAGNETOSPHERE}

The alternation of the above processes leads to the fact that the outer electron radiation belt "breathes" all the time, and not only the intensity of fluxes of its inhabiting particles varies, but also dimensions of RB itself do. These variations are most pronounced in a quasi-trapping zone.

Electron flux measurements made by a geostationary satellite along with magnetic activity indices are exemplified in Figure 4, in which we show several characteristic variations of electron fluxes. A rapid quasiharmonic variation with a period of $24 \mathrm{hr}$ (the orbital period) is associated with the day-night asymmetry of the magnetosphere. Besides the rapid adiabatic decrease in electron flux intensity during the storm main phase (1), we see intervals with a slow decrease in intensity (2) during periods of low substorm activity and, conversely, of its slow increase (3), which is not induced by momentary activity but by the outward gradient radial drift from deeper RB regions (3).

These variations are generally short-lived, flux intensity decreases are compensated by increases, and the average monthly RB remains stable. At the same time, there may be long-term depletions of the radiation belt, which have been discussed in a number of papers. Thus, in [Jaynes et al., 2014], a long-term decrease in RB electron flux is explained by the fact that in a quiescent period, without acceleration, all losses are attributed to $700 \mathrm{keV}-2 \mathrm{MeV}$ electron precipitation (up to $92 \%$ and more). Ukhorskiy et al. [2015], on the contrary, note that losses at the magnetopause are responsible for most of the losses at $L>5$, whereas at smaller $L$ the decrease is adiabatic. Even during years of minimum solar activity, the level of magnetic disturbances remains sufficient to mask the depletion of the outer $\mathrm{RB}$, and only in the particularly deep minimum of 2009 there was a significant depletion [Kataoka, Miyoshi, 2010; Li et al., 2011; Lee et al., 2013; Lazutin, 2017]. Magnetic activity in those years was indeed very low, as derived from the distribution of the number of days with high and low $K_{\mathrm{p}}$ indices.

Figure 7 shows that there are much more intervals of a slow electron flux decrease, and of course they coin- 


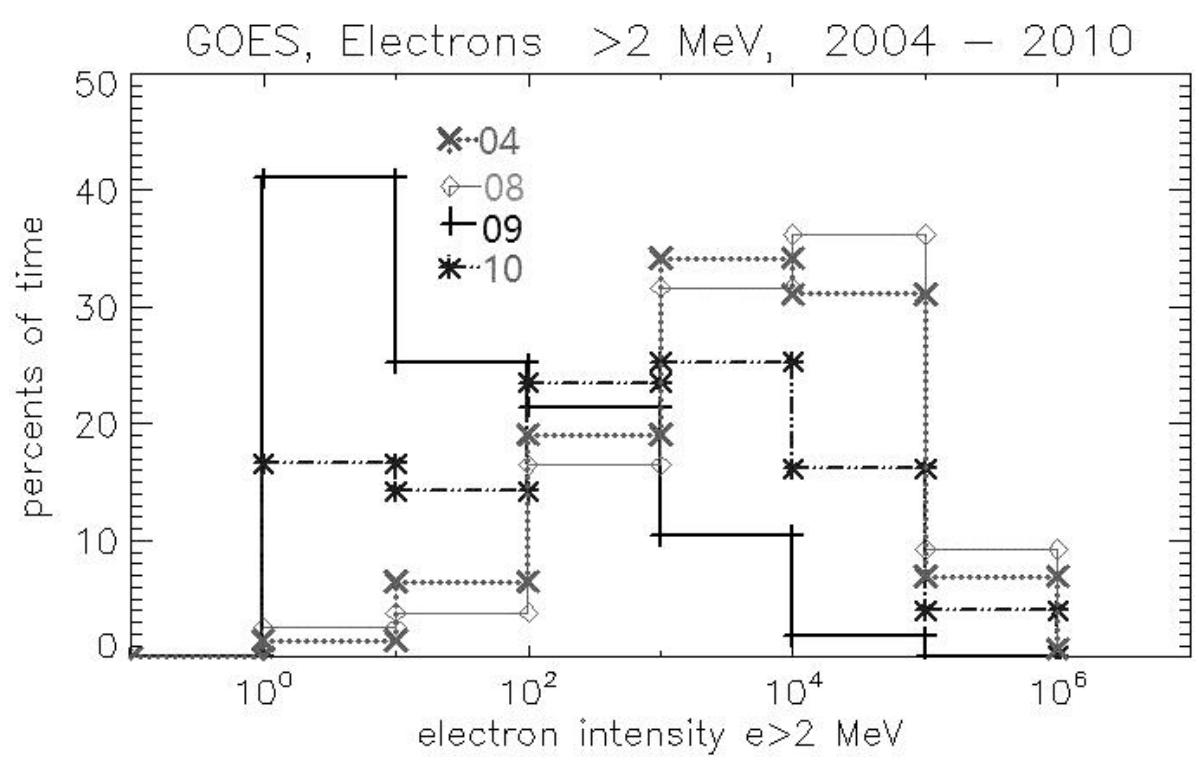

Figure 8. Distribution of electron flux levels in years of maximum (2004) and minimum (2008-1010) solar activity

cide with periods of low substorm activity. Finally, the depletion of RB is detected by comparing histograms of distribution of days with different electron flux levels in 2009 with years of higher magnetic activity (Figure 8). We mean the depletion of the entire RB because a decrease in the particle flux on the periphery of RB leads, due to diffusion, to the depletion of RB as a whole.

\section{CONCLUSION}

At the time free from magnetic disturbances (substorms), the outer electron radiation belt gradually empties. This process occurs on inner shells of RB due to precipitation of particles into the atmosphere and in a quasi-trapping zone because electron drift shells are not closed there. This causes losses at the magnetopause. The quasi-trapping region does not empty immediately due to radial diffusion of particles outward from the center of RB. The depletion is not well pronounced in ordinary years due to replenishment by freshly accelerated particles, but in years of extremely low activity, it leads to a considerable decrease in the electron population of the belt.

During the storm main phase, the major mechanism for the decrease in the intensity of electron fluxes is adiabatic cooling associated with conservation of adiabatic invariants. An additional reason is the precipitation of electrons from RB to the atmosphere and magnetopause. As a result, the intensity of electron fluxes in $\mathrm{RB}$ decreases. The $L$ coordinate calculated for the undisturbed configuration of the magnetospheric magnetic field is often used in plots of low-orbit satellite measurements. In these cases, we should remember that this representation does not show the real drift trajectory.

The rate of electron flux recovery after a storm is determined by the ratio of increases and losses, and flux values constitute a continuous series from low to very high values.

The electron flux increases consist of the following processes:
- The fast $\mathbf{E} \times \mathbf{B}$ electron shift, accompanied by an energy increase, deep to Earth by a pulsed induction electric field of substorm activation during a magnetic storm.

- This abrupt increase in the auroral electron flux intensity serves as the basis for the second, slower increase in the electron flux in RB due to electron injection to Earth by a large-scale electric field of the solar wind. A chain of several storms can sometimes increase the electron flux by several orders of magnitude, and this overpopulation of RB may decrease for months. In addition, there may appear additional RBs in a slot region.

On the periphery of $\mathrm{RB}$, in the quasi-trapping region, an increase in particle fluxes is ensured by the anti-earthward energetic electron drift, which replenishes the quasi-trapping region (geostationary region) with a 2-3 day delay.

The electron flux intensity in RB decreases mainly due to losses at the magnetopause, which become more intense during the storm main phase due to the adiabatic shift of drift shells and during periods of low substorm activity. During the extremely low activity of 2009, this mechanism led to significant losses of RB particles.

The resonance of electrons (cyclotron, drift) with VLF emission and pulsations leads both to their pitchangle diffusion to the loss cone and their dropout in the atmosphere, and to an increase in the energetic electron flux in parasitic resonance at the emission produced by a large flux of less energetic particles.

The combination of these processes forms the individual character of radiation belt dynamics during each magnetic storm.

\section{REFERENCES}

Anderson K.A. Balloon measurements of $\mathrm{X}$ rays in the auroral zone. Auroral Phenomena. Ed. M. Walt. Stanford University Press, Stanford, California, 1965, pp. 46-83.

Antonova E.E. Magnetospheric substorms and the sources of inner magnetosphere particle acceleration. The Inner Magnetosphere: Physics and Modeling. 2005, pp. 105-111. (Geophys. Monograph Ser., Vol. 155). DOI: 10.1029/155GM12. 
Bakhareva N.F. Nonstationary statistical acceleration of relativistic particles and their role during magnetic storms. Geomagnetizm i aeronomiya [Geomagnetism and Aeronomy]. 2003, vol. 43, pp. 737-744. (In Russian).

Baker D.N., Li X., Turner N., Allen J.H., Bargatze L.F., Blake J.B., Sheldon R.B., Spence H.E., Belian R.D., Reeves G.D., Kanekal S.G., Klecker B., Lepping R.P., Ogilvie K., Mewaldt R.A., Onsager T., Singer H.J., Rostoker G. Recurrent geomagnetic storms and relativistic electron enhancements in the outer magnetosphere: ISTP coordinated measurements. $J$. Geophys. Res. 1997, vol. 102, pp. 14,141-14,148. DOI: 10.1029/ 97JA00565.

Califf S., Li X., Zhao H. The role of the convection electric field in filling the slot region between the inner and outer radiation belts. J. Geophys. Res. 2017, vol. 122, pp. 20512068. DOI: 10.1002/2016JA023657.

Claudepierre S.G., Reeves G.D., O’Brien T.P., Fennell J.F., Blake J.B., Clemmons J.H., Looper M.D., Mazur J.E., Roeder J.L., Turner D.L. The hidden dynamics of relativistic electrons $(0.7-1.5 \mathrm{MeV})$ in the inner zone and slot region. $J$. Geophys. Res. 2017, vol. 122, pp. 3127-3144. DOI: 10.1002/ 2016JA023719.

Daibog E.I., Kechkemeti K., Lazutin L.L., Logachev Yu.I., Surova G.M. Relativistic electrons in the Earth's tail at the solar activity minimum. Izvestiya RAN. Seriya Fizicheskaya [Bulletin of the Russian Academy of Sciences: Physics]. 2015, vol. 79, no. 5, pp. 701-703. DOI: 10.7868/S036767651 5050191. (In Russian).

Demekhov A.G., Trakhtengerts V.Yu., Rycroft M.J., Nunn D. Electron acceleration in the magnetosphere by whistler-mode waves of varying frequency. Geomagnetizm i aeronomiya [Geomagnetism and Aeronomy]. 2006, vol. 46, no. 6, pp. 711-716. (In Russian).

Dmitriev A.V., Chao J.-K. Dependence of geosynchronous relativistic electron enhancements on geomagnetic parameters. J. Geophys. Res. 2003, vol. 108, pp. 1388. DOI: 10.1029/2002JA009664.

Dmitriev A.V., Jayachandran P.T., Tsai L.-C. Elliptical model of cutoff boundaries for the solar energetic particles measured by POES satellites in December 2006. J. Geophys. Res. 2010, vol. 115, A12244. DOI: 10.1029/2010JA015380.

Dmitriev A.V., Suvorova A.V., Chao J.K., Wang C.B., Rastaetter L., Panasyuk M.I., Lazutin L.L., Kovtyukh A.S., Veselovsky I.S., Myagkova I.N. Anomalous dynamics of the extremely compressed magnetosphere during 21 January 2005 magnetic storm. J. Geophys. Res. 2014, vol. 119, iss. 2, pp. 877-896. DOI: 10.1002/2013JA019534.

Elkington S.R., Hudson M K., Chan A.A. Acceleration of relativistic electrons via drift-resonant interaction with toroidal-mode Pc-5 ULF oscillations. Geophys. Res. Lett. 1999, vol. 26, iss. 21, pp. 3273-3276. DOI: 10.1029/1999GL003659.

Foster J.C., Erickson P.J., Omura Y., Baker D.N., Kletzing C.A., Claudepierre S.G. Van Allen Probes observations of prompt $\mathrm{MeV}$ radiation belt electron acceleration in nonlinear interactions with VLF chorus. J. Geophys. Res. 2017, vol. 122, pp. 324-339. DOI: 10.1002/2016JA023429.

Friedel R.H.W., Reeves G.D., Obara T. Relativistic electron dynamics in the inner magnetosphere - a review. $J$. Atmos. Solar-Terr. Phys. 2002, vol. 64, iss. 2, pp. 265-282. DOI: https://dx.doi.org/10.1016/S1364-6826(01)00088-8.

Gabrielse C., Harris C., Angelopoulos V., Artemyev A., Runov A. The role of localized inductive electric fields in electron injections around dipolarizing flux bundles. J. Geophys. Res.: Space Phys. 2016, vol. 121, pp. 9560-9585. DOI: 10.1002/2016JA023061.

Gabrielse C., Angelopoulos V., Harris C., Artemyev A., Kepko L., Runov A. Extensive electron transport and energization via multiple, localized dipolarizing flux bundles. $J$.
Geophys. Res.: Space Phys. 2017, vol. 122, pp. 5059-5076. DOI: 10.1002/2017JA023981.

Horne R.B., Thorne R.M. Potential waves for relativistic electron scattering and stochastic acceleration during magnetic storms. Geophys. Res. Lett. 1998, vol. 25, iss. 15, pp. 30113014. DOI: 10.1029/98GL01002.

Horne R.B., Thorne R.M. Relativistic electron acceleration and precipitation during resonant interactions with whistler mode chorus. Geophys. Res. Lett. 2003, vol. 30, iss. 10, 1527. DOI: 10.1029/2003GL016973.

Horne R.B., Thorne R.M., Glauert S.A., Albert J.M., Meredith N.P., Anderson R.R. Timescale for radiation belt electron acceleration by whistler mode chorus waves. J. Geophys. Res. 2005, vol. 110, A03225. DOI: 10.1029/2004JA010811.

Hudson M.K., Baker D.N., Goldstein J., Kress B.T., Paral J., Toffoletto F.R., Wiltberger M. Simulated magnetopause losses and Van Allen Probe flux dropouts. Geophys. Res. Lett. 2014, vol. 41, pp. 1113-1118. DOI: 10.1002/2014GL059222.

Hwang J., Choi E.-J., Park J.-S., Fok M.C., Lee D.Y., Kim K.C., Shin D.K., Usanova M.E., Reeves G.D. Comprehensive analysis of the flux dropout during 7-8 November 2008 storm using multisatellite observations and RBE model. J. Geophys. Res. 2015, vol. 120, iss. 6, pp. 4298-4312. DOI: 10.1002/2015JA021085.

Ivanova T.A., Pavlov N.N., Rezman S.Ya., Rubinshtein I.A., Sosnovets E.N., Tverskaya L.V. Dynamics of the outer radiation belt of relativistic electrons in the solar activity minimum. Geomagnetizm i aeronomiya [Geomagnetism and Aeronomy]. 2000, vol. 40, no. 1, pp. 13-18.

Jaynes A.N., Li X., Schiller Q.G., Blum L.W., Tu W., Turner D.L., Ni B., Bortnik J., Baker D.N., Kanekal S.G., Blake J.B., Wygant J. Evolution of relativistic outer belt electrons during an extended quiescent period. J. Geophys. Res. 2014, vol. 119, iss. 12, pp. 9558-9566. DOI: 10.1002/20 14JA020125.

Jaynes A.N., Baker D.N., Singer H.J., Rodriguez J.V., Loto'aniu T.M., Ali A.F., Elkington S.R., Li X. Kanekal S.G., Fennell J.F., Li W., Thorne R.M., Kletzing C.A., Spence H.E., Reeves G.D. Source and seed populations for relativistic electrons: their roles in radiation belt changes. J. Geophys. Res. 2015, vol. 120, iss. 9, pp. 7240-7254. DOI: 10.1002/2015 JA021234.

Kabin K., Kalugin G., Donovan E., Spanswick E. Particle energization by a substorm depolarization. J. Geophys. Res. 2017, vol. 122, pp. 349-367. DOI: 10.1002/2016JA023459.

Kalegaev V.V., Vlasova N.A. The Earth's magnetosphere response to interplanetary medium conditions on January 2122, 2005 and on December 14-15, 2006. Adv. Space Res. 2014, vol. 54, pp. 517-527. DOI: 10.1016/j.asr.2013.11.015.

Kalegaev V.V., Vlasova N.A., Peng J. Magnetosphere dynamics during 21-22.01.2005 and 14-15.12.2006 magnetic storms. Kosmicheskie issledovaniya [Cosmic Reseach]. 2015, vol. 53, no. 2, pp. 105-117. DOI: 10.7868/S002342061502003X. (In Russian).

Kataoka R., Miyoshi Y. Why are relativistic electrons persistently quiet at geosynchronous orbit in 2009? Space Weather . 2010, vol. 8, S08002. DOI: 10.1029/2010SW000571.

Kim H.J., Chan A.A. Fully adiabatic changes in stormtime relativistic electron fluxes. J. Geophys. Res. 1997, vol. 102, p. 22,107. DOI: 10.1029/97JA01814.

Kim K.C., Lee D.-Y., Kim H.-J., Lyons L.R., Lee E.S., Oztürk M.K., Choi C.R. Numerical calculations of relativistic electron drift loss effect. J. Geophys. Res. 2008, vol. 113, A09212. DOI: 10.1029/2007JA013011.

Kubota Y., Omura Y. Rapid precipitation of radiation belt electrons induced by EMIC rising tone emissions localized in longitude inside and outside the plasmapause. J. Geophys. Res. 2017, vol. 122, pp. 293-309. DOI: 10.1002/2016JA023267. 
Kuznetsov S.N. The behavior of the outer radiation belt of the Earth according to satellites Electron-1 and Electron-2. Izvestiya RAN. Seriya Fizicheskaya [Bulletin of the Russian Academy of Sciences: Physics]. 1966, vol. 30, iss. 11, pp. 18291837. (In Russian).

Kuznetsov S.N., Lazutin L.L., Panasyuk M.I., Starostin L.I., Gotseliuk Yu.V., Hasebe N., Sukurai K., Hareyama M. Solar particle dynamics during magnetic storms of July 23-27, 2004. Adv. Space Res. 2009, vol. 45, iss. 4, pp. 553-558. DOI: 10.1016/ j.asr.2008.09.014.

Lazutin L.L. On radiation belt dynamics during magnetic storm. Adv. Space Res. 2012, vol. 49, no 2, pp. 302-315. DOI: 10.1016/j.asr.2011.09.015.

Lazutin L.L. Dawn-dusk asymmetry and adiabatic dynamic of the radiation belt electrons during magnetic storm. Adv. Space Res. 2016, vol. 58, iss. 6, pp. 897-902. DOI: 10.1016/j. asr.2016.05.047.

Lazutin L.L. Depletion of the outer radiation belt during low activity years. Adv. Space Res. 2017, vol. 59, iss. 9, pp. 2248-2254. DOI: 10.1016/j.asr.2017.02.008.

Lazutin L.L., Panasyuk M.I., Hasebe N. Accelerations and losses of energetic protons and electrons during August 30-31, 2004 magnetic storm. Cosmic Res. 2011, vol. 49, no. 1, pp. 35-41.

Lazutin L.L., Logachev Yu.I., Muravieva E.A., Petrov V.L. Relaxation of electron and proton radiation belts of the Earth after strong magnetic storms. Kosmicheskie issledovaniya [Cosmic Reseach]. 2012, vol. 50, no. 1, pp. 3-14. (In Russian).

Lazutin L.L. Injection of relativistic electrons into the internal magnetosphere during magnetic storms: connection with substorms. Geomagnetizm i aeronomiya [Geomagnetism and Aeronomy]. 2013, vol. 53, no. 6, pp. 716-732. DOI: 10.7868/S0016794013050118. (In Russian).

Lee D.-Y., Shin D.-K., Kim J.-H., Cho J.-H., Kim K.-C., Hwang, J.A., Turner D.L., Kim T.K., Park M.-Y. Long-term loss and reformation of the outer radiation belt. J. Geophys. Res.: Space Phys. 2013, vol. 118, pp. 3297-3313. DOI: 10.1002/ jgra.50357.

Li X., Roth I., Temerin M., Wygant J.R., Hudson M.K., Blake J.B. Simulations of the prompt energization and transport of radiation belt particles during the March 24, 1991 SSC. Geophys. Res. Lett. 1993, vol. 20, p. 2423.

Li X., Baker D.N., Temerin M., Cayton T.E., Reeves G.D., Christensen R.A., Blake J.B., Looper M.D., Nakamura R., Kanekal S.G. Multisatellite observations of the outer zone electron variation during the November 3-4, 1993, magnetic storm. J. Geophys. Res. 1997, vol. 102, iss. A7, pp. 14,12314,140. DOI: 10.1029/97JA01101.

Li X., Temerin M., Baker D.N., Reeves G.D., Larson D. Quantitative prediction of radiation belt electrons at geostationary orbit based on solar wind measurements. Geophys. Res. Lett. 2001, vol. 28, iss. 9, pp. 1887-1890. DOI: 10.1029/ 2000 GL012681.

Li X., Temerin M., Baker D.N., Reeves G.D. Behavior of $\mathrm{MeV}$ electrons at geosynchronous orbit during last two solar cycles. J. Geophys. Res. 2011, vol. 116, A11207. DOI: 10.1029/2011JA016934.

Logachev Yu.I., Lazutin L.L. On the belt of energetic electrons at $L=2.75$ in the Earth's magnetosphere. Cosmic Res. 2012, vol. 50, no. 2, p. 116.

Matsumura C., Miyoshi Y., Seki K., Saito S., Angelopoulos V., Koller J. Outer radiation belt boundary location relative to the magnetopause: Implications for magnetopause shadowing. J. Geophys. Res. 2011, vol. 116, A06212. DOI: 10.1029/2011J A016575.

McIlwain C.E. Ring current effects on trapped particles. J. Geophys. Res. 1966, vol. 71, p. 3623.

Meredith N.P., Horne R.B., Glauert S.A., Anderson R.R. Slot region electron loss timescales due to plasmaspheric hiss and lightning generated whistlers. J. Geophys. Res. 2007, vol. 112, A08214. DOI: 10.1029/2007JA012413.
Millan R.M., Baker D.N. Acceleration of particles to high energies in Earth's radiation belts. Space Sci. Rev. 2012, vol. 173, iss. 1-4, pp. 103-131. DOI: 10.1007/s11214-012-9941-x.

Millan R.M., Thorne R.M. Review of radiation belt relativistic electron losses. J. Atmos. Solar-Terr. Phys. 2007, vol. 69, pp. 362-377. DOI: 10.1016/j.jastp.2006.06.019.

Ni B., Xing Cao, Zhengyang Zou. Resonant scattering of outer zone relativistic electrons by multiband EMIC waves and resultant electron loss time scales. J. Geophys. Res. 2015, vol. 120, iss. 9, pp. 7357-7373. DOI: 10.1002/2015JA021466.

Nishida A. Outward diffusion of energetic particles from the Jovian radiation belt. J. Geophys. Res. 1976, vol. 81, p. 1771.

Parks G.K., Winkler J.R. Acceleration of energetic electrons observed at the synchronous altitude during magnetospheric substorms. J. Geophys. Res. 1968, vol. 73, p. 5786.

Pavlov N.N., Tverskaya L.V., Tverskoy B.A., Chuchkov E.A. Radiation belt particle flux changes during strong magnetic storm of March 24, 1991. Geomagnetizm i aeronomiya [Geomagnetism and Aeronomy]. 1993, vol. 33, no. 6, pp. 41-45. (In Russian).

Potapov A.S. Relativistic electrons of the outer radiation belt and their predictions (review). Solnechno-zemnaya fizika [Solar-Terr. Phys.]. 2017, vol. 3, no. 1, pp. 46-58. DOI: 10.12737/22210. (In Russian).

Reeves G.D., Baker D.N., Belian R.D., Blake J.B., Cayton T.E., Fennell J.F., Friedel R.H.W., Meier M.M., Selesnick R.S., Spence H.E. The global response of relativistic radiation belt electrons to the January 1997 magnetic cloud. Geophys. Res. Lett. 1998, vol. 17, iss. 25, p. 3265. DOI: 10.1029/98GL02509.

Reeves G.D., McAdams K.L., Friedel R.H.W., O’Brien T.P. Acceleration and loss of relativistic electrons during geomagnetic storms. Geophys. Res. Lett. 2003, vol. 30, iss. 10, p. 1529. DOI: 10.1029/2002GL016513.

Saito S., Miyoshi Y., Seki K. A split in the outer radiation belt by magnetopause shadowing: test particle simulations. $J$. Geophys. Res. 2010, vol. 115, A08210. DOI: 10.1029/2009JA 014738.

Shprits Y.Y., Thorne R.M., Friedel R., Reeves G.D., Fennell J., Baker D.N., Kanekal S.G. Outward radial diffusion driven by losses at magnetopause. J. Geophys. Res. 2006, vol. 111, A11214. DOI: 10.1029/2006JA011657.

Shprits Y.Y., Elkington S., Meredith N.P., Subbotin D.A. Review of modeling of losses and sources of relativistic electrons in the outer radiation belt I: radial transport. J. Atmos. Solar-Terr. Phys. 2008a, vol. 70, pp. 1679-1693. DOI: 10.1016/j.jastp.2008. 06.008.

Shprits Y.Y., Subbotin D.A., Meredith N.P., Elkington S. Review of modeling of losses and sources of relativistic electrons in the outer radiation belt II: local acceleration and losses. J. Atmos. Solar-Terr. Phys. 2008b, vol. 70, pp. 1694-1713. DOI: 10.1016/j.jastp.2008.06.014.

Simms L.E., Engebretson M.J., Pilipenko V., Reeves G.D., Clilverd M. Empirical predictive models of daily relativistic electron flux at geostationary orbit: multiple regression analysis. $J$. Geophys. Res. 2016, vol. 121, pp. 3181-3197. DOI: 10.1002/ 2016JA022414.

Slivka M., Kudela K., Kuznetsov S.N. Some aspects of relativistic electron fluxes dynamics in the outer radiation belt during magnetic storms. Acta Physica Slovaca. 2006. vol. 56, no. 2, pp. 103-107.

Summers D., Thorne R.M. Relativistic electron pitchangle scattering by electromagnetic ion cyclotron waves during geomagnetic storms. J. Geophys. Res. 2003. vol. 108, iss. A4, p. 1143. DOI: 10.1029/2002JA009489.

Summers D., Ma C., Mukai T. Competition between acceleration and loss mechanisms of relativistic electrons during geomagnetic storms. J. Geophys. Res. 2004, vol. 109, A04221. DOI: 10.1029/2004JA010437. 
Summers D., Thorne R.M., Xiao F. Relativistic theory of waveparticle resonant diffusion with application to electron acceleration in the magnetosphere. J. Geophys. Res. 1998, vol. 103, iss. A9, pp. 20487-20500. DOI: 10.1029/98JA 01740.

Tang C.L., Zhang J.-C., Reeves G.D., Su Z.P., Baker D.N., Spence H.E., Funsten H.O., Blake J.B., Wygant J.R. Prompt enhancement of the Earth's outer radiation belt due to substorm electron injections. J. Geophys. Res.: Space Phys. 2016, vol. 121, pp. 11,826-11,838. DOI: 10.1002/2016JA023550.

Turner D.L., Shprits Y., Hartinger M., Angelopoulos V. Explaining sudden losses of outer radiation belt electrons during geomagnetic storms. Nat. Phys. 2012, vol. 8, pp. 208212. DOI: $10.1038 /$ nphys2185.

Turner D.L., O’Brien T.P., Fennell J.F S. Claudepierre G., Blake J.B., Jaynes A.N., Baker D.N., Kanekal S., Gkioulidou M., Henderson M.G., Reeves G.D. Investigating the source of nearrelativistic and relativistic electrons in Earth's inner radiation belt. J. Geophys. Res. 2017, vol. 122, pp. 695-710. DOI: 10.1002/2016JA023600.

Tverskaya L.V., Ivanova T.A., Pavlov N.N., Reizman S.Ya., Rubinstein I.A., Sosnovets E.N., Veden'kin N.N. Storm-time formation of a relativistic electron belt and some relevant phenomena in other magnetospheric plasma domains. Adv. Space Res. 2005, vol. 36, pp. 2392-2400. DOI: https://doi.org/10. 1016/j.asr.2003.09.071.

Tverskoi B.A. Transfer and acceleration of charged particles in the magnetosphere of the Earth. Geomagnetizm i aeronomiya [Geomagnetism and Aeronomy]. 1965, vol. 5, pp. 793-809. (In Russian).

Tverskoi B.A. Osnovy teoreticheskoi kosmofiziki [Foundations of Theoretical Space Physics]. Moscow, URSS Publ., 2004. 376 p.

Tverskoi B.A. Dynamics of the Earth's radiation belts. Geomagnetizm i aeronomiya [Geomagnetism and Aeronomy] 1964, vol. 4, pp. 436-448. (In Russian).

Ukhorskiy A.Y., Anderson B.J., Takahashi K., Tsyganenko N.A. The impact of ULF oscillations in the solar wind dynamic pressure on the outer radiation belt electrons. Geophys. Res. Lett. 2006, vol. 33, iss. 6, L06111. DOI: 10.1029/2005 GL024380.

Ukhorskiy A.Y., Sitnov M.I., Millan R.M., Kress B.T., Fennell J.F., Claudepierre S.G., Barnes R.J. Global storm time depletion of the outer electron belt. J. Geophys. Res. 2015, vol. 120, iss. 4, pp. 2543-2556. DOI: 10.1002/2014JA020645.

Vampola A.L., Korth A. Electron drift echoes in the inner magnetosphere. J. Geophys. Res. 1992, vol. 19, iss. 6, pp. 625628. DOI: 10.1029/92GL00121.

Vernov S.N., Gorchakov E.V., Kuznetsov S.N., Logachev Yu.I., Sosnovets E.N., Stolpovs V.G. Particle fluxes in the outer geomagnetic field. Rev. Geophys. 1969, vol. 7, no. 12 , pp. 257-280.
Vernov S.N., Chudakov A.E., Vakulov P.V., Gorchakov Ye.V., Kuznetsov S.N., Logachev Yu.I., Nikolaev A.G., Rubinshtein I.A., Sosnovets E.N., Stolpovsky V.G., El'tekov V.A. Results of the investigation of radiation belt particle position and energy based on satellite Electron-1 and Electron-2 measurements. Space Investigations. Moscow, Nauka Publ., 1965, pp. 394-405. (In Russian).

Xiao F., Chang Yang, Zhaoguo He, Zhenpeng Su, Qinghua Zhou, Yihua He, Kletzing C.A., Kurth W.S., Hospodarsky G.B., Spence H.E., Reeves G.D., Funsten H.O., Blake J.B., Bake D.N., Wygant J.R. Chorus acceleration of radiation belt relativistic electrons during March 2013 geomagnetic storm. J. Geophys. Res.: Space Phys. 2014, vol. 119, pp. 3325-3332. DOI: 10.1002/2014JA019822.

Yang X.C., Zhu G.W., Zhang X.X., Sun Y.Q., Liang J.B., Wei X.H. An unusual long-lived relativistic electron enhancement event excited by sequential CMEs. J. Geophys. Res.: Space Phys. 2014, vol. 119, p. 119. DOI: 10.1002/2014JA 019797.

Ying Xiong, Lun Xie, Zuyin Pu, Suiyan Fu, Lunjin Chen, Binbin Ni, Wen Li, Jinxing Li, Ruilong Guo, Parks G.K. Responses of relativistic electron fluxes in the outer radiation belt to geomagnetic storms. J. Geophys. Res. 2015, vol. 120, iss. 11, pp. 9513-9523. DOI: 10.1002/2015JA021440.

Yu J., Li L.Y., Cao J.B., Yuan Z.G., Reeves G.D., Baker D.N., Blake J.B., Spence H. Multiple loss processes of relativistic electrons outside the heart of outer radiation belt during a storm sudden commencement. J. Geophys. Res.: Space Phys. 2015, vol. 120, pp. 10,275-10,288. DOI: 10.1002/2015JA021460.

Zakharov A.V., Kuznetsov S.N. Electron precipitation and VLF emission. Geomagnetizm i aeronomiya [Geomagnetism and Aeronomy]. 1978, vol. 18, no. 2, pp. 352-353.

Zhenpeng Su, Hui Zhu, Fuliang Xiao, Huinan Zheng, Yuming Wang, Q.-G. Zong, Zhaoguo He, Chao Shen, Min Zhang, Shui Wang, Kletzing C.A., Kurth W.S., Hospodarsky G.B., Spence H.E., Reeves G.D., Funsten H.O., Blake J.B., Baker D.N. Quantifying the relative contributions of substorm injections and chorus waves to the rapid outward extension of electron radiation belt. J. Geophys. Res. 2014, vol. 119, iss. 12, pp. 10,023-10,040. DOI: 10.1002/2014JA020709.

How to cite this article

Lazutin L.L., Dmitriev A.V., Suvorova A.V. Electron radiation belt dynamics during magnetic storms and in quiet time. Solar-Terrestrial Physics. 2018, vol. 4, no. 1, pp. 51-60. DOI: 10.12737/stp-41201805. 